\title{
CALIDAD DE VIDA ASOCIADA AL GRADO DE SECUELA RADIOLÓGICA DE TUBERCULOSIS PULMONAR EN PACIENTES ATENDIDOS POR EL DEPARTAMENTO DE NEUMOLOGÍA DEL HOSPITAL NACIONAL HIPÓLITO UNÁNUE EN EL 2016
}

\author{
QUALITY OF LIFE ASSOCIATED WITH THE RADIOLOGICAL SEQUELAE OF \\ PULMONARY TUBERCULOSIS IN PATIENTS ATTENDED BY THE PNEUMONOLOGY \\ DEPARTMENT OF THE NATIONAL HOSPITAL HIPÓLITO UNÁNUE IN 2016 \\ Manuel Javier Swayne-Encalada', Patricia Segura-Nuñez 2,a,b,c, \\ Manuel Huamán-Guerrero 2,3,d,e
}

\begin{abstract}
RESUMEN
La tuberculosis es un gran problema de salud a nivel mundial. Causa problemas de salud entre millones de personas cada año y es la principal causa de morbilidad en muchas regiones, por ello comprender su efecto sobre la calidad de vida y estado de salud es importante para el cuidado del paciente. Objetivos: Identificar la asociación entre calidad de vida y afetación del parenquima pulmonar mediante grado de secuela radiológica de tuberculosis pulmonar en pacientes atendidos por el departamento de neumología del Hospital Nacional Hipólito Unánue en el 2016. Métodos: Estudio observacional, cuantitativo, analítico y transversal. Se encuestaron a 162 pacientes atendidos por el departamento de neumología con el antecedente de haber sido afectados por tuberculosis pulmonar en el Hospital Nacional Hipólito Unánue en el 2016. Se aplicó el test de calidad de vida SF-36 validado en el Perú por Salazar y Bernabé el 2012, además el grado de secuela radiológica clasificó según Willcox. Resultados: La correlación de Pearson fue de - 0.157 lo cual significa que hay una relación negativa entre calidad de vida y grado de secuela radiológica de tuberculosis pulmonar, además se halló significancia estadística ya que $\mathrm{P}=0.046(\mathrm{P}<0.05)$. Conclusión: Se comprueba asociación entre calidad de vida y grado de secuela radiológica de tuberculosis pulmonar pero ésta es una correlación negativa débil.
\end{abstract}

Palabras clave: Calidad de vida; Secuela radiológica; Tuberculosis pulmonar. (fuente: DeCS BIREME)

\begin{abstract}
Tuberculosis is a major global health problem. It causes health problems among millions of people every year and is the main cause of morbidity in many regions, so the understanding of its effect on quality of life and health status is important for patient care. Objective: Identify the association between quality of life and degree of radiological sequelae of pulmonary tuberculosis in patients treated by the neumology department of the National Hospital Hipolito Unanue in 2016. The Quality of Life test SF36 was applied, the test was validated by Salazar and Bernabé in 2012; in addition the degree of radiological sequelae is classified according to Willcox. Methods: CObservational, quantitative, analytical and transverse study. We surveyed 162 patients treated by the neumology department with the clinical antecedent of pulmonary tuberculosis in the National Hospital Hipolito Unanue in 2016. Results: Pearson's correlation was -0.157 , which means that there is a negative relation between quality of life and degree of pulmonary sequelae of pulmonary tuberculosis. Statistical significance was also found since $P=0.046(P<0.05)$. Conclusions: There is an association between quality of life and radiological sequelae of pulmonary tuberculosis, but this is a weak negative correlation.
\end{abstract}

Key words: Quality of life; Radiological sequelae; Pulmonary tuberculosis. (source: MeSH NLM)

\footnotetext{
1 Internado Medico, Hospital Nacional Hipolito Unanue.

2 Instituto de Investigación en Ciencias Biomédicas, Facultad de Medicina Humana, Universidad Ricardo Palma, Lima, Perú.

${ }^{3}$ Especialista en Cirugía Oncológica.

${ }^{a}$ Médico cirujano, ${ }^{b}$ Magíster en Medicina, ' Especialista en Neumología, d Doctor en Medicina.
}

Correspondencia: Manuel Javier Swayne Encalada. Dirección: Av. De las Artes Norte 252, San Borja; Lima, Perú. Teléfono: 2250303. Celular: 988890398. Correo: spinel_sun10@hotmail.com

Citar como: Manuel Javier Swayne-Encalada, Patricia Segura-Nuñez, Manuel Huamán-Guerrero. Calidad de vida asociada al grado de secuela radiológica de tuberculosis pulmonar en pacientes atendidos por el departamento de neumología del Hospital Nacional Hipólito Unánue en el 2016. [Artículo Original]. Rev. Fac. Med. Hum. 2017;17(1):26-31. DOI 10.25176/RFMH.v17.n1.745 


\section{INTRODUCCIÓN}

La tuberculosis (TB) es uno de los grandes problemas de salud a nivel mundial. Causa problemas de salud entre millones de personas cada año y ocupa junto con el virus de inmunodeficiencia humana $(\mathrm{VIH})$ la principal causa de muerte en el mundo'.

Según la OMS, en el año 2014, había un estimado 9,6 millones de nuevos casos de tuberculosis: 5,4 millones entre hombres, 3,2 millones entre las mujeres y 1 millón de niños. También se registraron muertes a causa de esta enfermedad: 1,5 millones de personas, de las que aproximadamente 890000 fueron hombres, 480000 eran mujeres y 140000 eran niños'.

La tuberculosis es la principal causa de morbilidad en muchas regiones, por ello comprender su efecto sobre la calidad de vida y estado de salud es importante para el cuidado del paciente y la evaluación de nuevos tratamientos o estrategias preventivas así como también para políticas de salud, como datos sobre la calidad de vida se utilizan en evaluaciones económicas de salud ${ }^{2}$.

El deterioro en la salud relacionado con la calidad de vida es un constructo complejo de influencias incluyendo el bienestar físico, mental y social ${ }^{3}$ - así la enfermedad que es experimentada por los individuos, no puede ser entendida de forma independiente en las sociedades en que viven ${ }^{4}$. Aunque una definición biológica de la infección (es decir, tuberculosis latente) o enfermedad (tuberculosis activa) puede ser universal, no es posible producir un único valor numérico que resume el impacto de la tuberculosis en un individuo. No es de extrañar, por lo tanto, que los estudios cuantitativos han reportado una amplia gama de valores para el deterioro de la salud asociadas con tuberculosis antes, durante y después del tratamiento ${ }^{5}$.

El Ministerio de la Protección Social señala que unos doce millones de colombianos están infectados por el bacilo de la tuberculosis. La incidencia anual de tuberculosis asciende hasta aproximadamente 10.000 en Colombia, y $15 \%$ de estos son aportados por el Departamento de Antioquia 6 .

Anualmente, en el Perú se notifican aproximadamente 27 mil y 17 mil casos nuevos de enfermedad activa y tuberculosis pulmonar frotis positivo, respectivamente, causando que seamos uno de los países con más altos casos de tuberculosis en América; una emergente aparición de cepas resistentes complica prevenir y controlarla, los anteriores dos años han habido más de 1500 pacientes con tuberculosis multidrogoresistente (MDR) al año y de 80 casos de tuberculosis extremadamente resistente (XDR) al año7.
El problema epidemiológico que trae la tuberculosis no solo afecta a nivel físico, sino también emocional y social. Debido a ser una patología de largo tratamiento implica compromiso y constancia para una curación adecuada; por la limitación física que se halla durante la primera etapa del tratamiento y en casos de fármaco resistencia, resultando en un déficit de la capacidad laboral que a su vez se refleja en menos ingresos económicos; dado que estos factores deterioraran la calidad de vida de las personas que padecen esta enfermedad ${ }^{8}$.

Los trabajos realizados acerca de la calidad de vida en pacientes con secuelas radiológicas de tuberculosis pulmonar son escasos. El Hospital Nacional Hipólito Unánue tiene considerable concurrencia de pacientes con tuberculosis pulmonar y que consecuentemente brinda seguimiento y tratamiento a pacientes con secuelas por tuberculosis, sería el lugar más adecuado para realizar el presente estudio.

\section{MÉTODOS}

Se realizó un estudio de tipo observacional, cuantitativo, analítico y transversal.

La población de estudio estuvo conformada por 812 pacientes atendidos por el departamento de neumología en el Hospital Nacional Hipólito Unánue en el 2016. En la presente investigación se consideró una muestra estratificada probabilística. Para calcular el tamaño mínimo de la muestra, y así obtener una muestra mínima representativa utilizó la fórmula de las proporciones obteniéndose una muestra de 162 personas, lo cual representa aproximadamente el $5 \%$ de la población y es la que se consideró para este estudio. Se dividió y reclutó de acuerdo al grado de secuela radiológica que se aprecia en el estudio de Di Naso, 2011. Para el grado I, 53 pacientes siendo este $38 \%$ de nuestra muestra, 24 y 66 siendo $17 \%$ y $45 \%$ respectivamente. La unidad de análisis fue un paciente atendido por el departamento de neumología con el antecedente de haber sido afectado por tuberculosis pulmonar en el Hospital Nacional Hipólito Unánue en el 2016. El instrumento utilizado fue el Test de calidad de vida SF 36 que ha sido validado en el estudio de Salazar y Bernabé del 2012 donde la confiabilidad de la consistencia interna fue estimada usando el coeficiente de a de Cronbach el cual tuvo una media de 0.82 y un rango entre 0.66 a 0.929 . Dicho cuestionario fue elaborado y estructurado por Medical Outcomes Study MOS (Estudio de resultados médicos) en estados unidos en la década de los 90 para la evaluación de calidad de vida, posteriormente siendo actualizado 
y traducido a diversos idiomas. Otro instrumento utilizado fue la clasificación según Willcox de grados de secuela radiológica de tuberculosis pulmonar, en donde el grado I es la afección mínima envolviendo a una zona sin cavitación, el grado II envuelve a dos o tres zonas, o zonas con cavitación, por último, el grado III envuelve a más de tres zonas con o sin cavitación. La clasificación de los grados se obtuvo la colaboración de en un neumólogo. Además, se utilizó una ficha de recolección de datos. Los datos fueron procesados utilizando el programa estadístico spss v22. Se utilizaron los estadísticos de correlación de Pearson y media para demostrar la asociación de calidad de vida y secuela de tuberculosis pulmonar.

\section{RESULTADOS}

Tabla 1. Prevalencia de datos sociodemográficos Parte 1.

\begin{tabular}{|lccc|}
\hline \multicolumn{1}{|c}{ Edad } & & Frecuencia & Porcentaje \\
\hline Total: 162 & Jóvenes & 0 & 0 \\
& Adultos & 146 & $90,1 \%$ \\
\hline & Adultos & 16 & $9,9 \%$ \\
& Mayores & & -- \\
\hline Género & Media & 42,67 & Porcentaje \\
\hline Total: 162 & Masculino & 93 & $57,4 \%$ \\
\hline \multirow{4}{*}{ Estado civil } & Femenino & 69 & $42,6 \%$ \\
\hline Total: 162 & Soltero & 62 & Frecuencia \\
\hline & Casado & 29 & $17,9 \%$ \\
\hline & Conviviente & 54 & $33,3 \%$ \\
\hline & Divorciado & 8 & $4,9 \%$ \\
\hline & Viudo & 8 & $4,9 \%$ \\
\hline & Unión Libre & 1 & $0,6 \%$ \\
\hline
\end{tabular}

Fuente: Ficha de Recolección de Datos.
Tabla 2. Prevalencia de datos sociodemográficos Parte 2.

\begin{tabular}{|c|c|c|c|}
\hline \multicolumn{2}{|c|}{ Grado de instrucción } & \multirow{2}{*}{$\begin{array}{c}\text { Frecuencia } \\
6\end{array}$} & \multirow{2}{*}{$\begin{array}{c}\text { Porcentaje } \\
\qquad 3,7 \%\end{array}$} \\
\hline Total: 162 & Analfabeta & & \\
\hline & $\begin{array}{l}\text { Primaria } \\
\text { Incompleta }\end{array}$ & 30 & $18,5 \%$ \\
\hline & $\begin{array}{l}\text { Primaria } \\
\text { Completa }\end{array}$ & 16 & $9,9 \%$ \\
\hline & $\begin{array}{l}\text { Secundaria } \\
\text { Incompleta }\end{array}$ & 40 & $24,7 \%$ \\
\hline & $\begin{array}{l}\text { Secundaria } \\
\text { Completa }\end{array}$ & 54 & $33,3 \%$ \\
\hline & Preuniversitario & 3 & $1,9 \%$ \\
\hline & $\begin{array}{l}\text { Universitario } \\
\text { Incompleto }\end{array}$ & 8 & $4,9 \%$ \\
\hline & $\begin{array}{l}\text { Universitario } \\
\text { Completo }\end{array}$ & 4 & $2,5 \%$ \\
\hline & Postgrado & 1 & $0,6 \%$ \\
\hline \multicolumn{2}{|c|}{ Comorbilidades } & Frecuencia & Porcentaje \\
\hline \multirow[t]{2}{*}{ Total: 162} & Presentes & 33 & $20,4 \%$ \\
\hline & Ausentes & 129 & $79,6 \%$ \\
\hline Ocupación & & Frecuencia & Porcentaje \\
\hline \multirow[t]{8}{*}{ Total: 162} & Estudiante & 22 & $13,6 \%$ \\
\hline & Ama de casa & 50 & $30,9 \%$ \\
\hline & Obrero & 40 & $24,7 \%$ \\
\hline & Empleado & 13 & $8,0 \%$ \\
\hline & Comerciante & 12 & $7,4 \%$ \\
\hline & Profesional & 1 & $0,6 \%$ \\
\hline & Pensionado & 4 & $2,5 \%$ \\
\hline & Desempleado & 20 & $12,3 \%$ \\
\hline
\end{tabular}

Fuente: Ficha de Recolección de Datos.

Tabla 3. Prevalencia de calidad de vida y grado de secuela radiológica de tuberculosis.

\begin{tabular}{llccccccc} 
& & \multicolumn{3}{c}{ Calidad de Vida } & & Total \\
& & Menor & & Adecuada & & Mayor \\
Grado de Secuela & Grado I & 12 & $19,67 \%$ & 19 & $31,15 \%$ & 30 & $49,18 \%$ & 61 \\
$\begin{array}{l}\text { Radiológica de } \\
\text { Tuberculosis }\end{array}$ & Grado II & 7 & $24,14 \%$ & 10 & $34,48 \%$ & 12 & $41,37 \%$ & 29 \\
\hline & Grado III & 17 & $23,61 \%$ & 30 & $41,66 \%$ & 25 & $34,72 \%$ & 72 \\
\hline Total & 36 & $22,22 \%$ & 59 & $36,42 \%$ & 67 & $41,36 \%$ & 162 \\
\hline
\end{tabular}

Fuente: Ficha de Recolección de datos y encuesta SF-36. 
Tabla 4. Asociación entre calidad de vida y grado de secuela radiológica de tuberculosis pulmonar.

\section{Calidad de vida}

\begin{tabular}{llc} 
Grado de secuela & Correlación de Pearson &,$- 157 "$ \\
Radiológica de Tuberculosis & Sig. (bilateral) &, 046 \\
& $\mathrm{~N}^{\circ}$ & 162 \\
\hline
\end{tabular}

Fuente: Ficha de Recolección de datos y encuesta SF-36.

Tabla 5. Prevalencia del grado de secuela radiológica de tuberculosis pulmonar.

\section{Frecuencia}

61

29

72

\section{Porcentaje}

$38 \%$

$18 \%$

$44 \%$

Grado III

Fuente: Ficha de Recolección de datos y encuesta SF-36.

Tabla 6. Promedios de las dimensiones de calidad de vida.

\begin{tabular}{cccccccc}
$\begin{array}{c}\text { Función } \\
\text { Física }\end{array}$ & Rol Físico & $\begin{array}{c}\text { Dolor } \\
\text { Corporal }\end{array}$ & $\begin{array}{c}\text { Salud } \\
\text { General }\end{array}$ & Vitalidad & $\begin{array}{c}\text { Función } \\
\text { Social }\end{array}$ & $\begin{array}{c}\text { Rol } \\
\text { Emocional }\end{array}$ & $\begin{array}{c}\text { Salud } \\
\text { Mental }\end{array}$ \\
\hline 72,38 & 32,72 & 63,73 & 40,67 & 58,02 & 72,15 & 48,35 & 62,44 \\
\hline
\end{tabular}

Fuente: Ficha de Recolección de datos y encuesta SF-36.

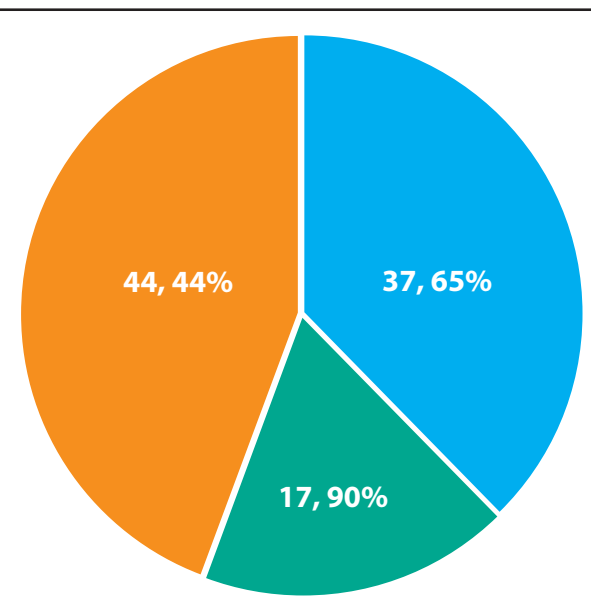

Grado de Secuela Radiologica de Tuberculosis -Gradol Grado II Grado III

Fuente: Ficha de Recolección de datos

Gráfico 1. Prevalencia del grado de secuela radiológica de tuberculosis.

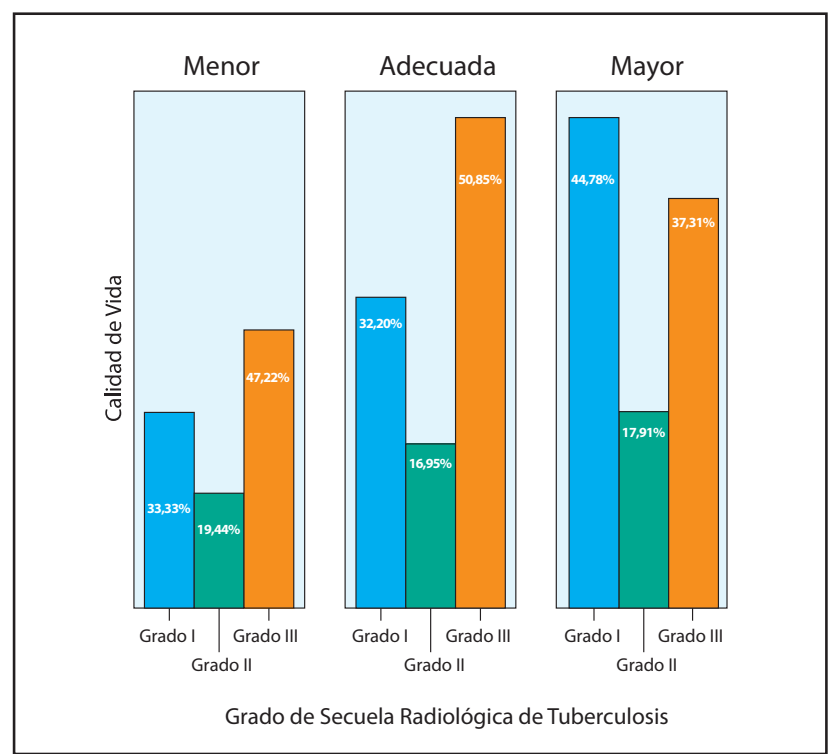

Fuente: Ficha de Recolección de datos

Gráfico 2. Calidad de vida según grado de secuela radiológica. 


\section{DISCUSIÓN}

Enesteestudiolaedadmediadelospacientesencuestados fue de 42,67 años. Que concuerda con el estudio de Bauer y cols del 2012 donde la edad promedio fue de 42 años, en cambio en el estudio de Loayza del 2016 el promedio se sitúa en 38 años probablemente siendo la causa la población estudiada y la pirámide poblacional de esta. Así también se halló que el grupo etario más afectado es el de adultos $90,1 \%$, estando acorde con las edades en los anteriores trabajos mencionados ${ }^{5,8}$. Además la gran parte de los encuestados fueron de género masculino con $57,4 \%$, encontrándose similitud con el estudio realizado por Loayza del 2016, donde señalan 56,25\% pertenecientes al género masculino, acorde también al análisis de situación epidemiológica de la tuberculosis en el Perú donde predominan los varones como género más afectado por la tuberculosis, así mismo el reporte global de tuberculosis del 2015 señala una diferencia de 2.2 millones de personas afectadas por tuberculosis siendo esta diferencia en favor del género masculino ${ }^{1,8,10}$. Así también se halló que la mayor parte de los encuestados son solteros siendo estos 38,3\% y conviviente en 33,3\%, a diferencia de estudio realizado por Loayza el 2016, donde la mayor parte de su muestra fue conviviente con $47,5 \%$ y soltero estuvo con $32,5 \%$, en el estudio realizado por Unalan y cols del 2008 en cambio la mayor parte de la unidad de análisis fue casado con $70,4 \%$, por lo que difieren entre poblaciones y momentos de ejecución de los trabajos ${ }^{8,11}$. El grado de instrucción que mayormente tuvieron los participantes se fue Secundaria siendo 58\%, se halla similitud con el estudio de Loayza del 2016 donde secundaria obtuvo el mayor porcentaje de grado de instrucción en sus resultados ${ }^{8}$. En relación a comorbilidades se encontró que la mayoría no las presentaba llegando a 79,6\%, lo cual también se observa en el trabajo de Loayza del 2016 con 85\% 8 . La ocupación más frecuente se encontró en ama de casa teniendo este $30,9 \%$, siendo gran parte de las mujeres desempleadas.

Encontramos que el grado I es conformado por más unidades de estudio en el grupo de mejor calidad de vida con 30 pacientes, el grado II viene a ser el grupo de mejor calidad de vida con 12 pacientes también, por último, en el grado III el grupo de calidad de vida adecuada con 30 pacientes. Es así que encontramos una relación perfecta negativa entre calidad de vida y grado de secuela radiológica de tuberculosis pulmonar con la correlación de Pearson en -0.157, siendo estadísticamente significativo ya que $\mathrm{P}=0.046(\mathrm{P}<0.05)$. Lo señalado anteriormente está en relación a la exclusión de los pacientes severamente afectados ya que ellos no podrían responder adecuadamente el Test de calidad de vida, por lo que la correlación podría cambiar.
Similar a la limitación funcional pulmonar y la calidad de vida en el trabajo de Fernández del 2011 donde a la disminución de la limitación funcional pulmonar se ve mejoría en la calidad de vida.

Se observar que el grado I de secuela radiológica de tuberculosis Pulmonar está representado por el 38\% de toda la muestra, el grado II por el $18 \%$ y el grado III por el $44 \%$. Lo cual es similar en el trabajo de Di Naso del 2011 que se hallan porcentajes similares siendo estos $38 \%, 17 \%$ y $45 \%$ respectivamente ${ }^{12}$.

En cuanto a resultados relacionados a las dimensiones de calidad de vida la función física obtuvo 72,38 puntos siendo este el promedio más alto entre las dimensiones, En comparación a otros estudios obtiene buenos resultados, pero no tanto como en este, estado entre la segunda a cuarta dimensiones siendo estos estudios Loayza del 2016, Fernández y cols del 2011 y Baltazar y cols 2013 respectivamente. En cambio, la dimensión con menor puntaje fue rol físico con 32,72 puntos, siendo respalda como la dimensión con menor puntaje en los estudios de Baltazar y cols del 2013, Fernández y cols 2011 difiriendo por una posición en el estudio de Loayza del 2016 siendo penúltima dimensión en cuanto a puntaje $\mathrm{e}^{8,13,14}$.

El grado de secuela radiológica de tuberculosis pulmonar con menor calidad de vida es la de Grado III con $47,22 \%$ de los pacientes con menor calidad de vida y la que tiene mayor calidad de vida es la de Grado I con $44,78 \%$ de los pacientes con mejor calidad de vida. Lo que se ve explicado en el trabajo de Fernandez del 2011 ya que mayor limitación funcional pulmonar mayor disminución de calidad de vida, ya que el grado de secuela radiológica está dado por el número de lesiones en zonas especificadas, a mayor limitación funcional pulmonar encontraremos mayor número de lesiones, ya sea en una misma zona o diferentes.

En esta investigación se encontraron algunas limitaciones como haber tomado el mínimo permitido para la muestra de la población estudiada. Además, al no ser posible manipular las variables solo se describen y se observa las asociaciones.

\section{CONCLUSIÓN}

Se identificó que hay una asociación entre calidad de vida y grados de secuela radiológica de tuberculosis pulmonar, pero esta es una correlación negativa débil; es estadísticamente significativo.

Se determinó que el grado de secuela radiológica de tuberculosis pulmonar más frecuente en pacientes atendidos por el departamento de neumología del Hospital Hipólito Unánue es el Grado III. 
Se observó además que la dimensión más afectada de calidad de vida en pacientes con secuela radiológica de tuberculosis pulmonar atendidos en el Hospital de estudio fue la de rol físico.

Por último, se halló que el grado de secuela radiológica de tuberculosis pulmonar asociado a la menor calidad de vida en pacientes atendidos en el hospital de estudio fue la de grado III.

\section{AGRADECIMIENTOS}

Al Dr. Jhony A. De La Cruz Vargas, Director Adjunto del INICIB-URP, por haber dirigido la Tesis y brindar toda la orientación para la presente publicación.
Financiamiento: Autofinanciado.

Conflicto de interés: Los autores declaran no tener conflictos de interés en la publicación de este artículo.

Recibido: 27 de febrero del 2017

Aprobado: 13 de marzo del 2017

\section{REFERENCIAS BIBLIOGRÁFICAS}

1. Tobergte DR, Curtis S. Global Tuberculosis Report. Vol. 53, Journal of Chemical Information and Modeling. 2013.

2. Brown J, CapocciS, Smith C, Morris S, Abubakar I, Lipman M. Health status and quality of life in tuberculosis. Int J Infect Dis [Internet]. 2015;32:68-75. Available from: http://dx.doi.org/10.1016/j.jjid.2014.12.045

3. WHO. Summary Report on Proceedings Minutes and Final Acts of the International Health Conference. Int Heal Conf [Internet]. 1946;(2):143. Available from: http://apps.who.int/iris/bitstream/10665/85573/1/ Official_record2_eng.pdf

4. Conrad P, Barker KK. The social construction of illness: key insights and policy implications. J Health Soc Behav. 2010;51(S):S67-79.

5. Bauer M, Leavens A, Schwartzman K. A systematic review and metaanalysis of the impact of tuberculosis on health-related quality of life. Qual Life Res. 2013;22(8):2213-35.

6. Alpi SV, Fernández H, Quiceno JM, Posada ML, Otalvaro C. Calidad de Vida Relacionada con la Salud y Apoyo Social Funcional en Pacientes Diagnosticados con VIH/Sida. Ter Psicológica [Internet]. 2008;26(1):12532. Available from: http://www.redalyc.org/articulo.oa?id=78526111

7. Internacional RS. Boletín Epidemiológico (Lima). 2013;22(21):455-73.

8. Loayza Adrianzen LG. Percepción de la calidad de vida en pacientes con tuberculosis pulmonar en segunda fase de tratamiento antituberculoso en el centro de salud El Agustino, noviembre - diciembre 2015. Universidad Ricardo Palma; 2016.

9. Salazar FR, Bernabé E. The Spanish SF-36 in Peru. Asia Pacific J Public Heal [Internet]. 2015;27(2):NP2372-NP2380. Available from: http:// journals.sagepub.com/doi/10.1177/1010539511432879

10. Soto Cabezas MG, Chavez Pachas AM. ANALISIS DE LA SITUACIÓN EPIDEMIOLOGICA DE LA TUBERCULOSIS EN EL PERÚ, 2015. Ministerio De Salud. Lima: Ministerio de Salud; 2015.

11. Unalan D, Soyuer F, Ceyhan O, Basturk M, Ozturk A. Is the quality of life different in patients with active and inactive tuberculosis? Indian J Tuberc. 2008;55(3):127-37.

12. Di Naso F.C., Pereira J.S., Schuh S.J. UG. Functional evaluation in patients with pulmonary tuerculosis sequelae. Port J Pulmonol. 2011;17(5):216-21.

13. Baltazar RG, Heredia AL. Calidad de vida relacionada con la salud y trabajo en pacientes con tuberculosis en Guadalajara. Salud Uninorte. 2013;29(1):13-21.

14. Fernández-Plata MR, García-Sancho MC, Pérez-Padilla JR. Impacto de la tuberculosis pulmonar en la calidad de vida de los pacientes. Un estudio de casos y controles. Rev Investig Clínica. 2011;63(1):39-45.

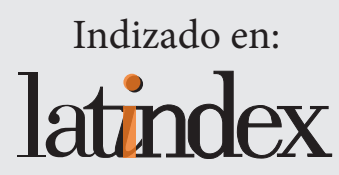

http://www.latindex.org/latindex/ficha?folio=14280

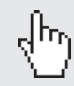

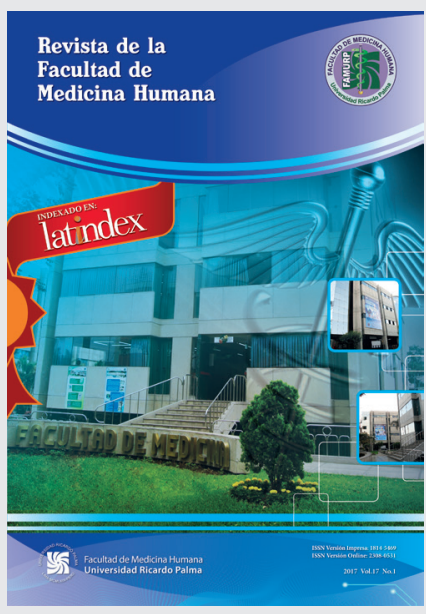

